\title{
INFRAESTRUCTURA Y COMPETITIVIDAD \\ EN LA INICIATIVA DE LA FRANJA Y LA \\ RUTA: IMPLICACIONES GEOECONÓMICAS \\ EN EL CASO DEL PUENTE HZM
}

Sxunasxi Marysol Valencia Crivelli(a)

Raquel I. León de la Rosa(b)

INFRASTRUCTURE AND COMPETITIVENESS IN THE BELT AND ROAD

INITIATIVE: GEOECONOMIC IMPLICATIONS IN THE CASE OF THE HZM BRIDGE

INFRAESTRUTURA E COMPETITIVIDADE NA INICIATIVA DE FAIXA E

ROTA: IMPLICAÇÕES GEOECONÔMICAS NO CASO DA PONTE HZM

Fecha de recepción: 26 de marzo del 2019

Fecha de aprobación: 30 de mayo del 2019

Disponible en línea: 28 de junio del 2019

Sugerencia de citación:

Valencia Crivelli, S.M. y León de la Rosa, R.I. (2019). Infraestructura y competitividad en la Iniciativa de la Franja y la Ruta: implicaciones geoeconómicas en el caso del puente HzM. Razón Crítica, 7, 81-110, doi: $10.21789 / 25007807.1499$

(a) Sxunasxi Marysol Valencia Crivelli

Comercio y logística internacional

Benemérita Universidad Autónoma de Puebla, México

https://orcid.org/0000-0002-5229-5619

sxunasxi.valencia@correo.buap.mx

(b) Raquel I. León de la Rosa

Estudios de China y relaciones transpacíficas

Benemérita Universidad Autónoma de Puebla, México

https://orcid.org/0000-0003-1019-0971

raquel.leon@correo.buap.mx 


\section{R E S U M E N}

El presente trabajo trata de la importancia que la infraestructura tiene dentro de la Iniciativa de la Franja y la Ruta. En específico, se enfoca en el caso del puente HZM como una de las más importantes obras que tienen un impacto en la actividad comercial de la región. Partiendo de esto, se busca determinar las ventajas a nivel comercial que la construcción este puente implica para la República Popular China (RPCH), en tanto que la construcción de este es una extensión de dicha iniciativa que trae grandes ventajas geoeconómicas a la RPCH hacia el exterior y también con respecto a sus regiones de administración especial.

PALABRAS CLAVE: comercio, geoeconomía, iniciativa OBOR, infraestructura, Hong Kong. 


\section{A B S T R A C T}

This work addresses the importance of infrastructure projects within the Belt and Road Initiative. Specifically, it focuses on the case of the HZM Bridge as one of the most important facilities with a direct impact on the commercial activity of the region. From this, we seek to determine the commercial advantages of the construction of this bridge for the People's Republic of China (PRC), since this engineering project is an extension of the Belt and Road Initiative that brings great geoeconomic advantages for this country with respect the rest of the world and its special administration regions.

KEYWORDS: овоR Initiative, infrastructure, trade, geoeconomics, Hong Kong.

\section{R E S U M O}

O presente trabalho aborda a importância la infraestrutura dentro da Iniciativa de Faixa e Rota. Especificamente, foca-se no caso da Ponte HZM como uma das mais importantes obras que têm um impacto na atividade comercial da região. Partindo disto, procura-se determinar as vantagens no nível comercial que a construção desta ponte implica para a República Popular China (RPCH), dado que a construção desta é uma extensão de dita iniciativa. Este projeto traz grandes vantagens geoeconômicas à RPCH no exterior, bem como a respeito de suas regiões de administração especial.

PALAVRAS-CHAVE: Iniciativa овоR, infraestrutura, comércio, geoeconomia, Hong Kong. 


\section{N T R O D U C C I Ó N}

En la política comercial de la República Popular China (RPCH), la Iniciativa de la Franja y la Ruta (BRI por sus siglas en inglés) ha ocupado un lugar clave con respecto a los desafíos de la convulsionada economía global. Pareciera que esta estrategia del presidente Xi Jinping avanza de una manera que le permite a China fortalecer su papel en distintas regiones del mundo. Esto, a su vez, le permite fortalecer su dinámica comercial y las relaciones con sus principales socios comerciales a través de redes que faciliten actividades económicas como la importación, la exportación y la inversión. La estrategia le permite a China, inclusive, ir más allá en los esquemas de cooperación tradicionales a través de los proyectos que impulsa.

En la función de la BRI dentro del actual posicionamiento internacional chino, se destaca uno de sus elementos clave: la infraestructura. Este artículo está destinado a analizar el caso más reciente en este ámbito: la construcción del Puente Hong Kong-Zhuhai-Macao, considerado hasta ahora el más largo del mundo. No obstante, más allá de esta discusión, la intención del análisis presentado es identificar las oportunidades para la RPCH a partir de la geoeconomía, rescatando dos aspectos: el incremento de la competitividad a partir de la facilitación del comercio y las implicaciones políticas de esta obra dentro de las regiones de administración especial, principalmente Hong Kong.

El siguiente aparte nos ofrece la idea de geoeconomía que guía este texto:

La penetración de los mercados con la ayuda del Estado reemplaza las bases y las guarniciones militares desplegadas en el extranjero, así como 
la influencia diplomática. Estas actividades representan las actividades cotidianas de las empresas privadas que trabajan por motivos meramente comerciales. Pero cuando el Estado interviene, cuando anima, asiste o dirige estas mismas actividades, no es ya sólo economía sino geoeconomía. (Luttwak, 1995, p. 42).

Partiendo de esta idea, se rescata el papel del Estado y la infraestructura como elementos que dinamizan el comercio con base en esta directriz que el gobierno chino ha dado para el incremento de la competitividad de la región y el beneficio de las empresas chinas a través de hubs. La ubicación geográfica y el estatus que tienen Hong Kong y Macao, como regiones de administración especial, ${ }^{1}$ son aspectos estratégicos importantes y únicos, pues representan la puerta de entrada a China continental.

Para abordar este tema, el presente artículo está conformado por una breve revisión histórica de la región del delta del río Perla en el contexto de la economía china, con la cual se vincula al objeto de estudio con la Iniciativa de la Franja y la Ruta. En este apartado se delimita geográficamente el espacio conformado por el delta. Después, se rescatan algunos conceptos teóricos vinculados con la competitividad para enlazarlos con el proyecto del Puente Hong Kong-Zhuhai-Macao, esto con el objetivo de dar paso a las siguientes secciones, en las que se revisa la potencialidad de este proyecto a partir de un antes y un después de su inauguración. Por último, se establecen una serie de conclusiones sobre el impacto económico del puente para la RPCH y las regiones de administración especial, principalmente Hong Kong,

Esta investigación es de índole descriptiva y exploratoria, ante la falta de análisis del objeto desde la perspectiva de geoeconomía y las variables propuestas. Las investigaciones o trabajos académicos hasta este momento se vinculan más con el área de ingeniería. No obstante, con respecto a la Iniciativa de la Franja y la Ruta y la vinculación con las regiones de administración especial, la principal información a rescatar corresponde a informes del gobierno de Hong Kong y de la RPCH. También se encuentran

1 Una región de administración especial es una división administrativa local establecida y reconocida por la Constitución de la RPCH. Las regiones de administración especial disfrutan, dentro de la división administrativa de China, de un estatus legal especial. Practican además el sistema capitalista dentro de su estilo de vida como una solución pacífica para el particular proceso histórico de Hong Kong, Macao y Taiwán hacia la formación del nuevo sistema socialista unitario de China (Zhou, 2013, p. 57). 
reportes económicos de instituciones como el Foro Económico Mundial (WEF por sus siglas en inglés) y Hong Kong and Shanghai Banking Corporation (HSBC). En la academia, la literatura más relevante que vincula el tema de la Iniciativa de la Franja y la Ruta con infraestructura y Hong Kong es la investigación de Roger Chan titulada "The Belt and Road Initiative: Opportunities for Developing Hong Kong's Infrastructure”. En esta investigación se plantea la importancia de este puerto como "superconector", entendido como un hub de transporte gracias a su conectividad e infraestructura. La principal conclusión de Chan es que este rol incrementa la competitividad de Hong Kong, insertado en la Iniciativa de la Franja y la Ruta como un "super-conector" que facilita la cooperación transfronteriza (Chan, 2017).

A partir de esta literatura, este artículo pretende ir más allá a través de la inclusión del concepto de geoeconomía como elemento que enlaza la competitividad, la infraestructura y el posicionamiento chino a nivel económico en las regiones de administración especial.

\section{Contexto del delta del río Perla y de la Iniciativa de la Franja y la Ruta en la economía china}

Se le llama delta del río Perla (Zhujiang) a la región sureña de China que abarca nueve megaciudades (Guangzhou, Shenzhen, Zhuhai, Foshan, Jiangmen, Zhong-shan, Jiangmen, Huizhou y Dongguan) y abarca el $30 \%$ de territorio de la provincia de Guandong (Liu, 2011). La figura 1 delimita a cada una de las ciudades y su ubicación.

Históricamente, el delta del río Perla ha desempeñado un papel muy importante a nivel comercial, principalmente en las dinastías Ming y Qing. Un primer momento de la importancia histórica de este delta se refiere al "periodo de comercio internacional precolonial", que tiene su lugar de 1450 a 1680 dentro del sistema tributario chino y sus rutas comerciales. Los puertos sureños eran un lugar estratégico para la consolidación del comercio exterior chino, el aseguramiento de fronteras y la paz en el Mar del Sur de China (Wolters, citado por Min, 2015). No obstante, cabe mencionar que durante este lapso ocurrió la instalación de los portugueses en Macao, en 1557, momento en el que este puerto tuvo bastante protagonismo. Después, vendría el periodo de comercio colonial, en el que el establecimiento de colonias europeas en el sureste asiático y la firma de tratados desiguales entre China y las potencias europeas y 


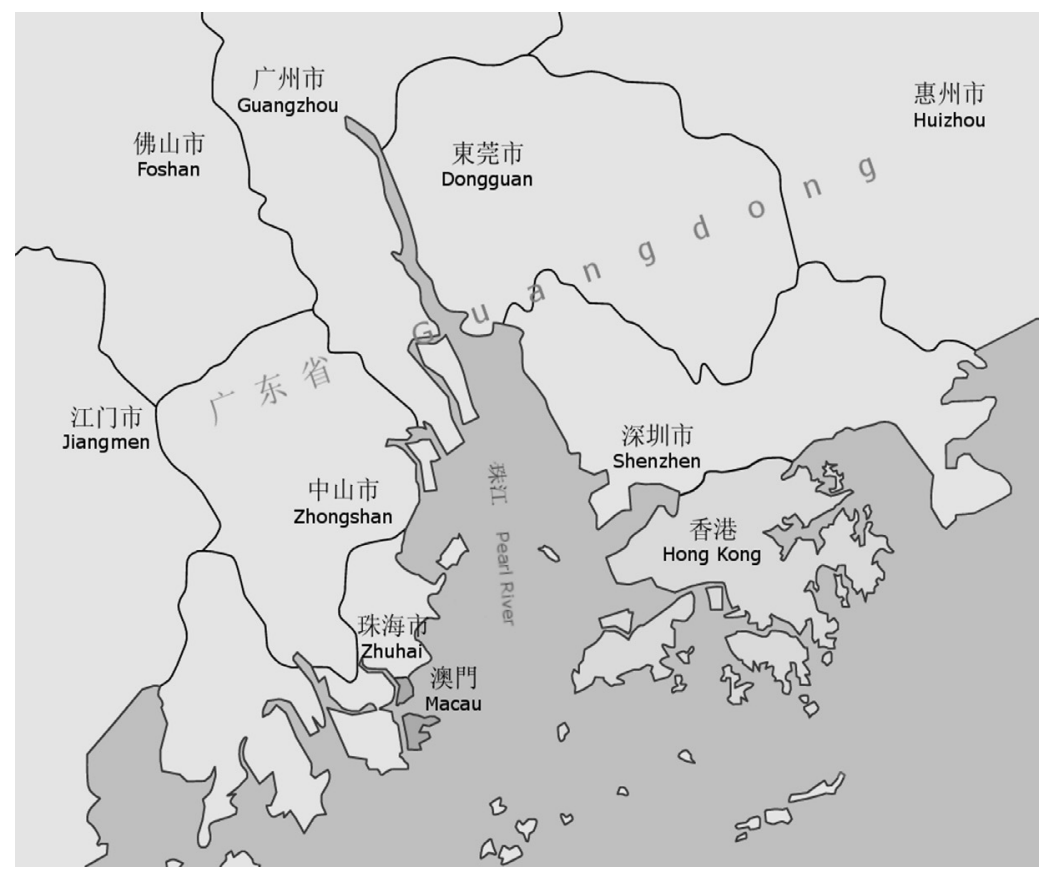

Figura 1. Delta del río Perla-Zhujiang.

Fuente: Wikipedia, 2007.

Japón generaron una nueva dinámica comercial en la región. Durante este periodo, la colonia británica en Hong Kong, tras el Tratado de Nanjing en 1842, determinó una serie de cambios en la dinámica de la región, lo cual significó un predominio de Hong Kong como el puerto con mayor actividad. Ante esta situación, se dio un momento de declive de Macao y del poderío mercante lusitano y, al mismo tiempo, se presentaron una serie de cambios administrativos en Guangzhou, declarado como puerto de acceso comercial entre 1684 y 1717 (Reid, 1996).

La continuidad de la presencia europea en Macao y Hong Kong conllevó a que cada uno tuviera su proceso histórico e importancia durante el siglo xx. Macao fue un territorio sin afectaciones durante la Segunda Guerra Mundial debido a su postura de neutralidad, mientras que Hong Kong fue uno de los principales puntos de la guerra en el Pacífico. No obstante, China Continental se sumergió durante este lapso en el proceso que puso fin al periodo imperial y produjo la confrontación entre nacionalistas y comunistas que, a su vez, dio origen a la RPCH. Varios años después, se dio un nuevo momento de auge para el delta del río Perla con el proceso de apertura económica china. La estrategia de 
implementación de Zonas Económicas Especiales (ZEE) que impulsaran este proceso provocó grandes cambios en el comercio exterior chino. En de la primera etapa del establecimiento de las zEe, la provincia de Guangdong fue la que más zonas tuvo. De acuerdo con Edith Papp, hubo una razón a nivel geoeconómico ${ }^{2}$ para que esta región fuera la que iniciara con el pilotaje:

\begin{abstract}
Shenzen, Zhuhai, Shantou y Xiamen surgieron como proyectos piloto para la restructuración controlada de la economía china, para probar en condiciones de laboratorio la transición de la economía centralmente planificada a la economía de mercado [...] A pesar de su tamaño reducido, su ubicación en la costa este y su cercanía con Hong Kong, Macao y Taiwán, las hizo especialmente interesantes para atraer capital — primero de la diáspora china y luego desde Occidente- mientras el incremento de los costes laborales en dichos entornos dirigió el interés de los inversores extranjeros hacia las zEE-s nuevas en busca de oportunidades. (Papp, 2013, p. 4-5).
\end{abstract}

La ubicación de cada una de las zEe responde a una razón, ya que frente a Hong Kong está Shenzhen, y frente a Macao, Zhuhai (Cornejo, 1985). La estrategia fue impulsada entonces para el posicionamiento económico a través del encadenamiento productivo de la región, principalmente de Hong Kong y Taiwán. El resultado de las zeE fue el desarrollo de un modelo de negocios en el que "Taiwán recibía los pedidos, la producción se hacía en la parte continental de China y las ventas se realizaban en el extranjero" (Liu, 2011). Este modelo muestra la importancia de la diáspora china en la región, determinando la especialización de las zEE en cinco sectores: alimentario, textil, maquinaria, equipamiento eléctrico y electrónica y telecomunicaciones. La importancia de esta dinámica llevó a que el delta del río Perla se convirtiera en una "megarregión”, que es un área contigua visible desde satélites que puede considerarse como una unidad geográfica que impulsa la economía mundial (Florida, Gulden y Mellander, 2007). En el plano económico, esta megarregión logró una mayor vinculación con Hong

2 Se entiende por geoeconomía el análisis de estrategias de orden económico, decididas por los Estados en el marco de políticas que tienden a proteger su economía nacional o algunos aspectos bien identificados por esta, y buscan adquirir el dominio de tecnologías clave o conquistar algunos segmentos del mercado mundial relativos a la producción y a la comercialización de un producto o de una gama de productos (Lorot, 1999, p. 15). 
Kong, ya que más del $70 \%$ de los acuerdos de inversión firmados en Guangdong entre 1976 y 2006 fueron con esta región de administración especial (Chen y Unteroberdoerster, 2008). Debido a esto, en el 2003, el secretario del Partido Comunista Chino en la provincia del Guangdong, Zhang Dejiang, propuso el término de "pan-región del delta del río Perla", promoviendo a su vez la estructura " $9+2$ ", que refiere a las nueve provincias vinculadas al río Perla ${ }^{3}$ y a las dos regiones de administración especial. De esta iniciativa surgió, en el 2003, la firma del "Acuerdo Marco de Cooperación Pan-regional del Delta del Río Perla” (Lin, s.f.)

Ante este escenario, fue necesario incrementar los proyectos de infraestructura para la facilitación del modelo de negocios. En 2008 se decidió conectar como una megaciudad a las nueve ciudades: Guangzhou, Shenzhen, Dongguan, Zhaoqing, Foshan, Huizhou, Jiangmen, Zhongshan y Zhuhai, a través de proyectos que fusionaran las redes de transporte, energía, agua y telecomunicaciones (Нsвсв, 2016). Esto permitió, al mismo tiempo, la consolidación de esta megarregión a través de clústeres. La tabla 1 muestra la especialización que las ciudades más representativas han tenido.

Tabla 1. Clústeres por ciudad dentro del Delta del Río Perla

\begin{tabular}{|c|c|}
\hline Ciudad & Clústeres \\
\hline Guangzhou & $\begin{array}{l}\text { Automóviles y autopartes, equipos de transporte, productos } \\
\text { eléctricos, electrónica, productos químicos, prendas de vestir, } \\
\text { textiles, servicios comerciales, software, juguetes. }\end{array}$ \\
\hline Shenzhen & $\begin{array}{l}\text { Electrónica, productos informáticos, productos de } \\
\text { telecomunicaciones, circuitos integrados, juguetes, plásticos, } \\
\text { relojes, pinturas al óleo, servicios portuarios, logística, finanzas, } \\
\text { impresión, árboles artificiales. }\end{array}$ \\
\hline Dongguan & $\begin{array}{l}\text { Electrónica, computadoras, componentes, periféricos, prendas de } \\
\text { vestir, muebles, zapatos, juguetes, relojes, cubiertos, utensilios de } \\
\text { cocina, máquinas de soldadura, equipos de pesca. }\end{array}$ \\
\hline Foshan & $\begin{array}{l}\text { Cerámica industrial, costura, textiles, } \\
\text { prendas para niños }\end{array}$ \\
\hline Huizhou & $\begin{array}{l}\text { Diodos láser, electrónica digital, CD-ROM, teléfonos, baterías, } \\
\text { circuitos, maquinaria de precisión, plásticos, productos químicos. }\end{array}$ \\
\hline Zhongshan & $\begin{array}{l}\text { Accesorios de iluminación, lámparas, productos de metal, } \\
\text { motocicletas, ropa, candados, equipos de audio. }\end{array}$ \\
\hline
\end{tabular}

Fuente: Chen \& Unteroberdoerster (2008, p. 6). 
El delta del río Perla tuvo un nuevo momento de auge por la implementación de las Zonas de Libre Comercio experimental (FTz por sus siglas en inglés) en el 2013: Guangzhou-Nansha, Shenzhen-QianhaiShekou y Zhuhai-Hengqin. El objetivo de estas zonas es incrementar la competitividad de la megarregión a través de la facilitación del comercio transfronterizo que, a su vez, permita el financiamiento en Renminbi (RMB) ${ }^{4}$ de dos vías entre la FTz y los dos centros offshore, es decir, Hong Kong y Macao, lo cual permite también un mayor posicionamiento de Shenzhen como clúster de innovación por la presencia de empresas como ISP, Tencent, zTE y Huawei, después de la aprobación de la Zona Nacional de Demostración de Innovación Independiente (ZNDII) en esta ciudad en el 2015. Esto ha permitido visibilizar una integración más contundente del delta del río Perla con Hong Kong y Macao, lo cual ha resultado en una contribución de más del $31 \%$ en la actividad comercial de Guangdong en el 2015, del cual el 24,6\% corresponde a Hong Kong. Al mismo tiempo, otro de los avances en la vinculación entre las FTz y las regiones de administración especial se muestra en el lanzamiento del programa Stock Connect de Shenzhen-Hong Kong a finales del 2016, el cual tiene el objetivo de atraer a más inversionistas globales a estas FTZ (HSBC, 2016).

El logro más reciente es la firma de una versión actualizada del Acuerdo de Asociación Económica más Estrecha (CEPA por sus siglas en inglés) entre China Continental y las regiones de administración especial en diciembre del 2018, con el objetivo de mejorar las reglas de origen para lograr la entrada de productos de las regiones con tasa cero. Al mismo tiempo, este acuerdo busca simplificar los procedimientos aduaneros, mejorar la transparencia de las medidas relacionadas con el comercio y fortalecer la cooperación en las áreas pertinentes. Una meta de suma importancia es acelerar el despacho de aduana de las mercancías, con el fin de facilitar el movimiento de mercancías en el área de la gran bahía de Guangdong-Hong Kong-Macao, lo cual mejorará la capacidad de despacho de aduanas y la eficiencia de los puntos de control en Guangdong y Hong Kong. Este acuerdo entró en vigencia el 1 de enero del 2019 (Gobierno de Hong Kong, 2018).

Con respecto a la BRI, existen seis corredores dentro de la propuesta: puente terrestre de Nueva Eurasia, China-Mongolia-Rusia, China-Asia 
Central-Asia Occidental, China-península Indochina, China-Pakistán y Bangladesh-China-India-Myanmar. Al mismo tiempo, la Ruta Marítima de la Seda surge como una iniciativa sumamente importante para consolidar el corredor indopacífico. Desde la academia, se identifica que estos corredores tienen una serie de objetivos e implicaciones (Kunaka, 2018):

- Incrementar la conectividad entre China y las comunidades económicas existentes.

- Fortalecer las redes de infraestructura.

- Generar acuerdos de cooperación en cada uno de los corredores. Aquí el Fondo de la Ruta de la Seda y el Banco Asiático de Inversiones en Infraestructura desempeñan un papel importante.

- Alineación de política de desarrollo por parte de las ciudades y pueblos parte de los corredores.

Dentro de la BRI existe una alineación con su impacto en el desarrollo económico de China, a partir de las estrategias de "Going out" y "Bringing In". Partiendo de estas estrategias, a las regiones de administración especial, principalmente a Hong Kong, se las ve como un enclave importante con respecto a la internacionalización del yuan (Wang, 2016). El gobierno de Hong Kong busca potencializar esta iniciativa a partir de los siguientes aspectos (Gobierno de Hong Kong, 2017):

- Ser un hub financiero y de servicios al comercio de impacto global.

- Su estatus político — "Un país, dos sistemas"- como una ventaja para la entrada comercial con China Continental.

Estos dos aspectos fortalecen cinco factores que hacen primordial la inserción de Hong Kong a la BRI: el Acuerdo de Asociación Económica más Estrecha (Closer Economic Partnership Arrangement, CEPA), condiciones de libre comercio, sistema legal robusto, ubicación geográfica e infraestructura avanzada (Gobierno de Hong Kong, 2017). En este contexto, el gobierno hongkonés ve la firma del acuerdo comercial entre Hong Kong y Georgia como el primer acuerdo insertado en el contexto de la BRI. Cabe destacar que desde la perspectiva pan-regional, con la firma del acuerdo marco en el 2003, existe una visión de conectividad principalmente con la región del sureste asiático (Lin, s.f.), lo cual se torna prioritario ante la existencia de estos corredores y los avances hacia la consolidación de la Asociación Regional Económica Integral (RCEP por sus siglas en inglés). Al mismo tiempo, no se debe dejar de lado la importancia de crear interdependencia económica entre China Continental y las regiones de administración especial en el proceso de reinserción. 


\section{El Puente HzM: competitividad y facilitación del comercio chino}

La consolidación de una macrorregión pujante de la economía asiática, así como su inserción en la BRI, representan innovación y oportunidades de crecimiento en términos de competitividad. De ahí que sea imperante indicar lo que para los países representa la competitividad, en el marco de un campo de investigación que es aún incipiente. Para explicar el impacto que el puente HzM ofrece a la macrorregión, se establecerá desde el inicio la noción de competitividad desde la perspectiva de los países.

Ante el nuevo escenario de economía y sociedad global, es importante destacar que el concepto de competitividad ha sido el punto central de las estrategias de los gobiernos y de la economía en general con el objetivo de generar productividad y diferenciación. Esta idea se ha visto soportada por instituciones internacionales, como el Consejo de la Agenda Global o el Foro Económico Mundial (wef por sus siglas en inglés). Este último, en su reporte sobre "Ciudades competitivas y sus conexiones con cadenas de valor globales", establece la importancia de la competitividad y el encadenamiento productivo internacional como dos conductores para un cambio exitoso de paradigmas en el siglo xxi y como motores de avances macrorregionales. Este reporte enfatiza los elementos políticos que pueden incrementar la participación de los países en redes internacionales a través de la innovación, lo que resultará en mayores índices de competitividad. A propósito de esto, el WEF define la competitividad de una ciudad como: "[...] el conjunto de factores (políticas, instituciones, estrategias y procesos) que determinan el nivel de productividad sostenible de una ciudad. La sostenibilidad abarca cuestiones económicas, ambientales y sociales" (WEF, 2016, p. 6).

El marco de referencia sobre los pilares de la competitividad de una ciudad, de acuerdo al WEF, se direcciona a: políticas y regulaciones, instituciones y conectividad. Tres de los pilares que se mencionan en el reporte del WEF incluyen la participación directa de los gobiernos, puesto que de ellos depende la integración de servicios que tienen impacto a nivel macroeconómico.

De ahí que el pilar sobre políticas y regulaciones centre al gobierno como actor preponderante en la toma de decisiones al momento de adecuar las políticas internas y externas con el fin de desarrollar proyectos que busquen mejorar la economía del país. Esto tiene un impacto directo 
en la competitividad del mismo gobierno en reformas políticas fiscales, comerciales, servicios, capital, trabajo, políticas económicas extranjeras, inversión extranjera directa, cadenas globales de valor, entre otros.

El siguiente pilar, respecto a las instituciones, se refiere a la forma de gobernanza en la toma de decisiones en referencia a la competitividad; es decir, tiene que ver con la implementación de iniciativas que busquen elevar los niveles de vida del país o la región: que sean proyectos innovadores y diferenciadores de la economía tradicional. Esto, sin duda alguna, marcará un nuevo aspecto de la competitividad moderna, la cual se puede lograr con la implementación de la tecnología. Empero, es importante que las instituciones busquen fortalecer lazos con la industria, la sociedad y otras instituciones que les ayuden a soportar dichos proyectos.

El último pilar, la conectividad en sus diferentes contextos, hace referencia directamente a la infraestructura con la que cuenta el país, y ofrece una clasificación de conectividad dura y suave. La primera se concentra en la conexión de las personas con los servicios básicos y los mercados, los diferentes medios de transporte, tecnologías de la información y comunicaciones, así como sistemas logísticos basados en clústeres de producción regional. Por su lado, la conectividad suave abarca el capital social y el capital del conocimiento, lo que hace más productivas las inversiones en infraestructura dura y nueva tecnología apoyando y complementando la innovación y el crecimiento económico. Cuando se implementen los dos tipos de conectividad se espera que las regiones presenten un crecimiento rápido, mejores y mayores empleos, y que estén preparadas para recibir y enviar Inversión Extranjera Directa (IED), así como que incrementen sus ingresos (WEF, 2016).

En referencia a lo mencionado, se reinterpreta el modelo de Michael E. Porter, "Determinantes de la Competitividad", para referirse a la competitividad en los siguientes términos:

Una nación o región es competitiva en la medida en que las empresas que operan en ella son capaces de competir de forma exitosa en la economía global y local a la vez que mantienen o incrementan los salarios y el estándar de vida de los ciudadanos, generando un espacio integrado y debidamente alineado en torno a una estrategia única y diferenciada (Azua, 2015, p. 3). 
El objetivo es crear un ecosistema espacial en el que las estrategias económicas y sociales se apliquen de manera convergente y simultánea en entornos clusterizados, más allá del concepto de "cadenas productivas o de valor" (Azua, 2015), complementado con el diamante competitivo, las cinco fuerzas, la clusterización y, según el concepto más reciente, la co-creación de valor-empresa-sociedad (shared value, en inglés) —concepto que aplica directamente a la relevancia e importancia de la BRI y el Puente HZM - Además de reactivar el flujo comercial en estas rutas, se espera que la BRI genere empleos para miles de personas y nuevas oportunidades de negocio para las empresas ubicadas en estas rutas. También será un centro de atracción de inversionistas de todo el mundo en áreas como infraestructura, finanzas, comercio, logística y servicios profesionales (HSBC, 2018).

El mismo Michael E. Porter pone en evidencia que algunas naciones y regiones son más poderosas que otras integrando en este proceso la innovación, no solo del sector privado, sino haciendo énfasis en un escenario global. Por lo tanto, un parteaguas de este crecimiento competitivo incluye ciertos factores determinantes que permiten elevar el nivel de competitividad de las regiones que se interconectan, a la vez que propician la agregación de economías bajo la conectividad inteligente (smart plugin, en inglés) de empresas, países y talento humano, coadyuvados por la tecnología pertinente. El éxito se consigue con el apoyo del gobierno, la sociedad y, adicionalmente, de las aportaciones empresariales, reafirmándolo con lo que menciona Azua: "Una explosión de ideas, nuevos paradigmas, en una economía cada vez más interrelacionada, más comprometida con la política en un nuevo espacio cooperativo empresa-empresa, región-región, empresa-gobierno y economía-sociedad” (Azua, 2015, p. 5).

De acuerdo con el Hong Kong Banking Report (KPMG, 2018), se espera que otras iniciativas regionales - como la Gran Área de la Bahía (GBA por sus siglas en inglés), que apunta a integrar económica y socialmente las nueve ciudades en el delta del río Perla de Guangdong, así como Hong Kong y Macao - transformen la región en un centro para facilitar la BRI. La combinación eficiente de capital, servicios financieros, tecnología e innovación y capacidades avanzadas de fabricación de las ciudades en la GBA continúa creando una base sólida para que las empresas de la región busquen oportunidades de salida a lo largo de la BRI.

Hong Kong se apoya enormemente en los servicios financieros, la producción de electrónica y el turismo. El sector agrícola es casi 
inexistente, ya que Hong Kong no procesa recursos naturales y depende completamente de importaciones de materias primas y energía. La contribución de la agricultura a la economía es prácticamente nula (0,07\% del PIB, y solo 0,21\% de la fuerza laboral en el 2017). La industria manufacturera representa una parte más amplia, pero aun así limitada, del Рів $(7,22 \%)$ y del empleo $(12,95 \%)$. Los principales sectores industriales del país son la electrónica, los electrodomésticos, la informática y las telecomunicaciones. El sector terciario representa el núcleo de la actividad económica: las industrias tradicionales clave en Hong Kong son los servicios financieros, comercio y logística, turismo, servicios profesionales y de producción. El sector terciario aporta $88,81 \%$ del PIB y emplea a cerca de $86,84 \%$ de la población activa. Hong Kong es un centro de servicios para las empresas asiáticas, especialmente para aquellas que tienen relaciones comerciales con China (Santander trade, 2019).

En el ámbito logístico, actualmente se ubica en el ranking de los cinco países con más registros de buques de capacidad de carga $\left(\mathrm{TPM}^{5}\right)$, al lado de Panamá, Liberia, las Islas Marshall y Singapur, con una cuota de mercado conjunta del 57,8\%. Igualmente, está en la quinta posición de los principales puertos del mundo, con un volumen de contenedores de 20 pies (TEU en inglés), en el 2016, de 19580 000; de la mano se encuentran los puertos de Guangzhou y Ningbo-Zhousan, los cuales reportaron una variación porcentual, del 2015 al 2016, de 8\% y 4,7\% (UNCTAD, 2017).

El modelo propuesto por Porter, y posteriormente complementado por Azua, encuentra aquí la coyuntura perfecta para la implementación de ambos proyectos (BRI y puente HZM): en la configuración de la megarregión encabezada por Hong Kong como punto logístico clave. Como hemos visto, este es uno de los puertos hub más eficientes del mundo, apoyado en su smart pluging e infraestructura como una red de negocios en transporte de carga marítima con una cobertura de 470 destinos internacionales y con la capacidad de recepción de buques portacontenedores de los clasificados como ultra-largos, aunado a la eficiencia aduanera, servicios de cadena de suministros (supply chain management en inglés) y aseguramiento de cargas. Cabe destacar que el crecimiento del puerto de Hong Kong ha sido resultado de la implementación del smart plugin del gobierno chino-industria naviera, con

5 Siglas de Tonelaje de Peso Muerto, unidad de medida de la capacidad máxima de carga de un buque cuando está cargado hasta su calado máximo admisible (incluyendo el material de rancho y la tripulación). 
la finalidad de promover el desarrollo a largo plazo de la región, tal como se representa hoy en día con la mega infraestructura del puente HzM, maximizando así la influencia de este en otras regiones de Asia-Pacífico a través de la intermodalidad carretera, marítima y aérea.

La culminación y puesta en marcha del puente HzM trae consigo beneficios de desempeño logístico-estratégicos, pues al ser una obra tan compleja de ingeniería y tecnología ofrece grandes ventajas económicas y sociales. El puente cuenta con una infraestructura que cruza el mar de Lingding, cuenta con 55 kilómetros de longitud, de los cuales 6,7 kilómetros son subterráneos (debido al tamaño de los buques que tienen rutas en esta zona), a través de los que se permite el movimiento de mercancías desde Zhuhai (en la parte continental), Macao, y se conecta el delta del río Perla hasta llegar a Hong Kong,

La interconectividad de la macrorregión no se focaliza únicamente en el puerto de Hong Kong, también se aprovechan las oportunidades que el Aeropuerto Internacional de Hong Kong (HKia por sus siglas en inglés) de esta ciudad ofrece con sus rutas internacionales en cuanto al movimiento de pasajeros y mercancías. El aeropuerto cubre más de 40 países de la BRI y se posiciona como el aeropuerto de carga número uno con un volumen de 5049898 toneladas métricas (ACI, 2019). Debido a esto se prevé una próxima expansión, derivada del incremento comercial, lo que consolidará a Hong Kong en una posición más importante a nivel global en términos logísticos. La figura 2 muestra la conectividad del puente HzM señalando como punto estratégico el HKIA.

En lo referente a transporte terrestre, por medio de carreteras y vías férreas, Hong Kong no se queda atrás, pues cuenta con la capacidad de desarrollo de vías innovadoras que soporten el proyecto de la BRI: vías para trenes de gran velocidad que cuenten con una operatividad sumamente exitosa.

Actualmente, en la sección de Hong Kong se encuentra el proyecto de "Guangzhou-Shenzhen-Hong Kong Express Rail Link (XRL)", el cual está en marcha y se espera dé inicio en el 2019. La finalidad de este proyecto es la conexión con la red ferroviaria de China Continental que se extiende a lo largo de $16000 \mathrm{~km}$. Este tren de alta velocidad traerá grandes beneficios, minimizando significativamente el tiempo de traslado entre Hong Kong y las principales ciudades de China vía férrea y, de esta manera, consolidará aún más la posición estratégica de Hong Kong. A la par, se desenvolverán y fortalecerán los lazos económicos de este con el continente e inyectará nuevo vigor en el crecimiento a mediano y largo 


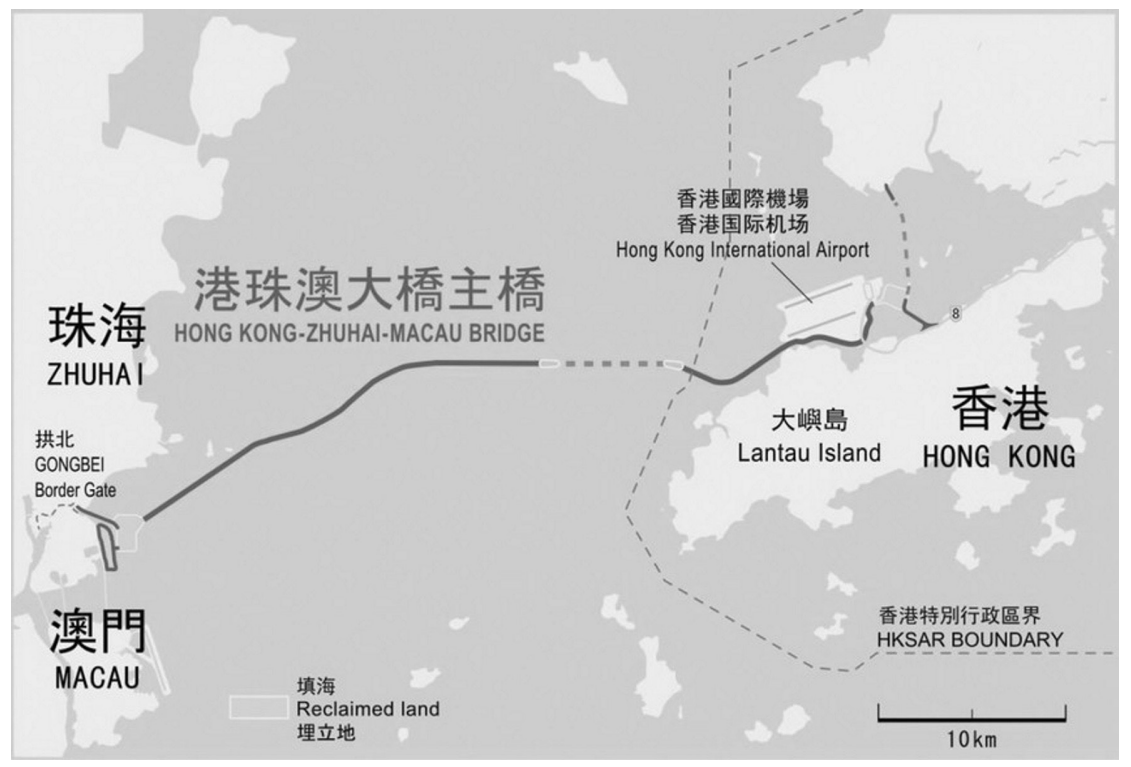

Figura 2. Conectividad puente HzM.

Fuente: WEF (2018).

plazo al crear nuevas oportunidades de negocios (Gobierno de Hong Kong, 2018).

\section{Infraestructura y comercio en el delta del río Perla previos a la construcción del puente}

La relación histórica entre Hong Kong y Macao (en el periodo comprendido entre 1949 y 1978) mostró algunos distanciamientos debido al proceso histórico que tuvieron como territorios bajo el dominio europeo y la instalación de la RPCH. Sin embargo, con la apertura económica de 1978 las relaciones comerciales se fortalecieron. Tras la llegada de Deng Xiaoping se establecieron tres zeE en Guangdong destinadas a explotar la cercanía de Hong Kong y Macao. Por su parte, Macao trasladó su industria textil a China mientras que Hong Kong movió sus procesos productivos a Guangdong, para posteriormente desarrollar una amplia base industrial en todos los sectores. Así fue como sus economías se fueron vinculando progresivamente hasta llegar a la integración con la que colaboran hoy en día. Finalmente, Guangdong se ha desarrollado gracias a las 
inversiones y el comercio con estas dos ciudades, aunque también hay que destacar la participación del gobierno chino con el aporte de grandes cantidades monetarias para la creación de la infraestructura necesaria para promover la IED y el comercio exterior. Este aporte se traduce en aeropuertos, carreteras, plantas de energía, alumbrado y electrificación, drenaje, centros educativos, hospitales, entre otros (Nava, 2012, p. 55). La permanente llegada de empresas extranjeras y el surgimiento de algunas locales han producido que la región del delta del río Perla arroje indicadores interesantes gracias a Hong Kong en primer plano. No obstante, es importante destacar el papel de cada uno de los tres territorios en la economía del río.

Las tres ZEE incentivaron la IED y con ello el incremento del intercambio comercial y el oportuno desarrollo de los puertos de la región que se vinculaban con Hong Kong, región administrativa que protagonizó la dirección de las exportaciones hacia China gracias a las industrias ahí establecidas (Nava, 2012). Las ZEE han ofrecido oportunidades de fabricación y de brindar servicios de comercialización de millones de mercancías con origen en Hong Kong, región que ha venido evolucionando gracias a su geolocalización y apertura al comercio, principalmente como centro de negocios hacia el sureste asiático.

Sin embargo, la relación con China no ha sido sencilla y empezó a fortalecerse solo con la aceptación en la Organización Mundial del Comercio (omc) tanto de Hong Kong en 1995 como de China en el 2001, pues los principios rectores de la omc respaldan la relación comercial que se favoreció y reforzó inicialmente con el cEPA. Por otro lado, la relación de Macao con China se estabilizó en 1999 cuando Macao fue devuelto por Portugal. Allí se desarrolló una industria ligera de algunas manufacturas, así como de casas de juegos y apuestas; en 1971, Macao se adhirió al General Agreement on Tariffs and Trade (GATT), antecesor de la омc, lo que le permitió la apertura a nuevas empresas extranjeras y el fortalecimiento del comercio mucho tiempo antes que otras regiones colindantes (Nava, 2012, p. 74).

La creación de las tres zeE en Guangdong, una de las cuales era la de Zhuhai, territorio colindante con Macao, facilitó que las empresas buscaran mover sus almacenes hacia esta región manteniendo sus oficinas en Macao, lo que resultó en empresas macaenses más rentables debido a los bajos costos que la provincia ofrecía (Nava, 2012, p. 75). En el 2003, cuando firma el CEPA con China, se enfatizan tres áreas económicas y comerciales: comercio de bienes, comercio de servicios y facilitación del 
comercio y la inversión. Actualmente, Macao es una de las macrorregiones económicamente más dinámicas de China y de todo el mundo. Posee todo tipo de empresas y conjuntos de empresas conformando los clústeres en sectores como el automotriz, electrodomésticos, calzado, juguetes, entre otros (Nava, 2012, p. 75).

La integración de la región de administración especial de Hong Kong con el continente, esencial para la transición de la ciudad a una economía basada en el financiamiento y el servicio, se ha producido principalmente en el delta del río Perla. Entre el 2001 y el 2006, el stock de IED hacia el exterior de la región de Hong Kong casi se triplicó a US \$270 mil millones y representó casi el $80 \%$ de toda la IED en el exterior. Se estima que el delta del río Perla capta alrededor del $40 \%$ de esta inversión. Las estadísticas provinciales de Guangdong indican que más del 70\% de todos los acuerdos de IED firmados entre 1979 y el 2006 son con compañías de la región administrativa especial de Hong Kong. Aproximadamente dos tercios de todas las importaciones de Hong Kong desde China Continental provienen de la provincia de Guangdong. Desde su apertura en 1979, la zona económica del delta del río Perla se ha convertido en el interior industrial, lo cual ha permitido a la región de administración especial de Hong Kong cambiar las fábricas a través de la frontera, aprovechar mano de obra industrial relativamente barata y aprovechar su ventaja en logística y otros servicios y finanzas para productores (Chen y Unteroberdoerster, 2008, p. 3).

La principal característica del delta del río Perla ha sido la promoción de la industria emergente y el servicio de clústeres, lo que ha permitido desarrollar una mayor especialización de la región, así como su adaptabilidad a cambios provenientes de la economía internacional. Por supuesto, fue necesario incluir la infraestructura adecuada específica para la región del delta del río Perla. Por su parte, la región administrativa especial de Hong Kong es uno de los territorios más urbanizados del mundo y cuenta con una excelente dotación de infraestructuras. Existían ya, antes de la construcción del HzM, tres túneles submarinos, nueve en superficie y tres puentes de gran escala, y el presupuesto destinado a carreteras para el período 2006-2012 fue de 3320 millones de euros (ICEX, 2010).

Guangdong, que poseía infraestructura precaria y poco desarrollada, necesitaba comenzar a desarrollar y modernizar su infraestructura para que este proceso de traslado de los procesos de producción, sumado a la llegada de otro tipo de IED, pudiera darse de forma exitosa. El gobierno 
chino comprendió este problema y, junto con capitales privados, comenzó a invertir para que Cantón (como también se le conoce a la región de Guangzhou) y otras provincias contaran con las condiciones adecuadas para su modelo de desarrollo económico. Las vías de comunicación fueron una de las áreas que más rápido se desarrolló en Cantón debido a su importancia para la llegada de nuevas empresas y la transportación de sus productos.

En 1980, la provincia de Cantón solo contaba con un sistema de vías férreas de 3206 km, un entramado de carreteras de 3967 km, había desarrollado solo $7887 \mathrm{~km}$ de 97 canales navegables, y poseía $143 \mathrm{~km}$ de un sistema de ductos. Después de diez años de inversión para el desarrollo, las vías férreas habían aumentado a $4803 \mathrm{~km}$, las carreteras a 63709, los canales navegables a $16198 \mathrm{~km}$ y el sistema de ductos a $1091 \mathrm{~km}$. Para el 2010, Guangdong llegó a contar con 18647 km de vías férreas, 125433 $\mathrm{km}$ de carreteras, $36623 \mathrm{~km}$ de canales navegables y un sistema de ductos de $6322 \mathrm{~km}$. Todo esto significa que en treinta años la longitud de las vías férreas creció cerca de $600 \%$, las carreteras crecieron más de $3100 \%$, los canales navegables se incrementaron $460 \%$, y el sistema de ductos creció 4400\% (Nava, 2012, pp. 97-98). Esto facilitó el comercio y brindó certidumbre a los inversionistas de la región. La población se vio beneficiada por la interconectividad de las ciudades teniendo acceso a un mercado mucho más vasto que el tradicional.

De manera generalizada, en el ámbito marítimo el comercio del transporte mundial ha venido desarrollándose gracias a la exigencia de las empresas, apoyadas por los gobiernos, de abastecerse de productos que generen diferenciación en costos e innovación. Esto ha traído consigo un aumento y una evolución del transporte: como señalan datos de la United Nations Conference on Trade and Development (UnCTAD) (2017, p. 1), "el volumen del comercio marítimo mundial creció un 2,6\% frente a un 1,8\% en 2015". En este contexto, China ha tenido un gran protagonismo; sin embargo, ha optado por una nueva política de consumo impulsado por su propio crecimiento interno mientras que anteriormente le apostaba más a las exportaciones.

En el Informe sobre el transporte marítimo en 2017, realizado por la UNCTAD (2017), se afirma que "las obras portuarias también son una característica destacada de la Iniciativa de la Franja y la Ruta. Varios países asiáticos como Malasia, Myanmar, el Pakistán y Sri Lanka han estado en la vanguardia de estos planes" (UnCTAD, 2017, p. 69). Aunado a esto, y debido a que las operaciones contenerizadas marítimas chinas corresponden a 
189285000 TEU's anualmente (UNCTAD, 2017), existe una congestión de las terminales portuarias y de distribución que afecta a otros países ubicados en Asia y altera las operaciones de enlace en la región. Los puertos de Shanghai, Qingdao y Ningbo han tenido problemas de congestión debido al aumento de los volúmenes, pero también como consecuencia de las redes de las alianzas navieras y la utilización de buques más grandes. Esto representa un enorme problema para las empresas navieras y logísticas ya que las operaciones en los puertos presentan retrasos y originan costos adicionales. La infraestuctura, hasta este momento, no tiene la capacidad para gestionar el volumen de TEU's, por lo que se requiere atender este punto por parte de la administración de puertos y el gobierno.

Como se muestra en la tabla 2, los puertos de Shenzhen, Hong Kong y Guangzhou presentan los siguientes volúmenes de TEU's manipulados en el período 2015-2016:

Tabla 2. Puertos de Shenzhen, Hong Kong y Guangzhou

\begin{tabular}{|c|c|c|c|c|}
\hline Puerto & Tráfico 2016 & Tráfico 2015 & $\begin{array}{c}\text { Variación } \\
\text { porcentual }\end{array}$ & Posición 2016 \\
\hline Shenzen & 23980000 & 24204000 & $-0,9$ & 3 \\
\hline Hong Kong & 19580000 & 20114000 & $-2,7$ & 5 \\
\hline Guangzhou & 18859000 & 17457000 & 8,0 & 7 \\
\hline
\end{tabular}

Fuente: Informe sobre transporte marítimo 2017, UNCTAD.

\section{Infraestructura y comercio en el delta del río Perla después de la construcción del puente}

En el 2009 comienza la construcción del puente HzM, proyecto sumamente ambicioso de China que busca una integración regional e internacional como parte del proyecto de la BRI. En esta iniciativa, la construcción del puente HzM cumple varios de los objetivos planteados de los cinco principales del proyecto (citado en KPMG, 2018):

1. Coordinación de políticas: colaboración entre varios países para resolver problemas y desarrollar soluciones de políticas que impulsarán el desarrollo social y económico a largo plazo de los países de la BRI.

2. Conectividad de las instalaciones: la priorización de la construcción debe ser compatible con la mejora del flujo 
de personas y bienes. Esto implica eliminar las barreras a la conectividad del transporte mediante el desarrollo de nuevas infraestructuras y mejoras a los enlaces existentes.

3. Comercio sin obstáculos: reducción de las barreras comerciales y aranceles, logrando menores costos de comercio e inversión y mejoras en las cadenas de suministro de la industria a través de las fronteras nacionales.

4. Integración financiera: mayor coordinación de la política monetaria de los países BRI, mejores sistemas locales de liquidación y cambio de moneda y mayor efectividad de los marcos financieros, regulatorios y de gestión de riesgos.

5. Lazos entre las personas: el fortalecimiento de las relaciones entre las personas de los países BRI y la promoción de una mayor conciencia y comprensión cultural.

En el tema financiero, se espera que la integración del Puente al proyecto BRI genere oportunidades para la provisión de otros productos de financiamiento e inversión. Un acuerdo de política firmado en diciembre del 2017 entre Hong Kong y la Comisión Nacional de Reforma del Desarrollo incluye un área de enfoque para garantizar que Hong Kong se adopte como una plataforma BRI para financiar soluciones tales como: financiamiento de infraestructura, bonos verdes y comercio de yuan (KPMG, 2018).

En el tema comercial, el objetivo del Puente es convertirse en una plataforma logística que brinde la conectividad necesaria para facilitar el comercio. La tecnología funge un papel importante en su construcción pues las condiciones geográficas del lugar en el que se localiza, así como la actividad comercial en esta región de Asia, obligan al desarrollo, investigación e innovación en muchos sentidos.

Lee afirma que:

El Consejo de Desarrollo del Comercio de Hong Kong ha calificado el proyecto de puente "de valor estratégico especial al consolidar el Área de la Bahía como la plataforma de servicios de inversión para la iniciativa Belt and Road y acelerar la integración económica e incrementar la competitividad regional”. (Lee, 2018, p. 2).

La gran inversión que los gobiernos de China y Hong Kong han hecho estima que el puente tenga como vida útil 120 años, favoreciendo de primera mano la macrorregión conformada por Macao-Zhuhai-Hong 
Kong. Pero la influencia se extiende a toda China a través del hinterland que se desarrolla con las ciudades que se encuentran en la parte occidental del delta.

El factor más importante en lo que respecta al impacto en el comercio asiático, con respecto a la infraestructura desarrollada, es el tiempo. La oportunidad de disminuir los traslados de Zhuhai a Hong Kong contribuye en eficiencia logística y minimización de costos, pues el tiempo estimado de traslado, después de la construcción del puente, es únicamente de una hora y media en promedio, lo cual proporciona un nuevo esquema de rutas para los actores del transporte internacional. Por ejemplo, antes de la construcción se tenía que, desde Zhuhai hasta la terminal de contenedores Kwai Tsing de Hong Kong, el tiempo de tránsito era de tres horas y media con una distancia de $200 \mathrm{~km}$; adicionalmente, desde el mismo origen hasta el HKIA el trayecto era de cuatro horas para la misma distancia. Con el puente HzM se reduce la distancia a $65 \mathrm{~km}$, correspondientes a 75 minutos, para arribar a la terminal portuaria y $40 \mathrm{~km}$, traducidos a 45 minutos, en el caso del нкіA. Los trayectos disminuyeron, así, más del $60 \%$ y $80 \%$, respectivamente (citado en Gere y Zoltai, 2018).

Los beneficios, comercialmente hablando, recaerán en un crecimiento del desempeño logístico, que contempla tiempos de tránsito y entrega de mercancías intrarregión, la conectividad de tipo hub en una intermodalidad que aprovecha la infraestructura con la que cuenta Hong Kong, mayores operaciones aduaneras que resultarán en más ingresos, así como la oportunidad de diversificar y descongestionar otros puertos de China agilizando el comercio. La IED también tendrá una fuerte presencia, pues será una actividad preponderante para extranjeros y locales, facilitando así el desarrollo de toda la estructura sectorial.

Sin embargo, a partir de su inauguración en octubre del 2018, los resultados aún tempranos de la utilización del Puente no apuntan a lo esperado. Actualmente, en el puente HzM se encuentra el Hong Kong Port, ubicado en un terreno reclamado de aproximadamente 130 hectáreas en las aguas del noreste del Aeropuerto Internacional de Hong Kong. A través de la red de transporte que incluye Hong Kong Link Road y Tuen Mun-Chek Lap Kok Link, las personas pueden viajar al puente principal de HzM, al Aeropuerto Internacional de Hong Kong, a Tuen Mun y al Norte de Lantau a través del Puerto de Hong Kong. También cuenta con dos edificios de inspección de rayos x para vehículos para fortalecer la capacidad de ejecución del Departamento de Aduanas e Impuestos Especiales. El sistema de inspección de rayos $\mathrm{x}$ del vehículo adopta un diseño tipo pórtico que 
es el primero de su tipo en Hong Kong. El sistema puede manejar dos contenedores de carga de 45 pies de largo al mismo tiempo en una sola inspección, con un rendimiento máximo de 20 camiones de contenedores por hora. Aplicando la tecnología de rayos x de transmisión de energía dual y la tecnología de rayos $\mathrm{x}$ de retrodispersión para inspeccionar vehículos y cargas, el sistema puede revelar de forma instantánea y clara los detalles de las mercancías dentro del contenedor, mostrándolas en diferentes colores de acuerdo con sus propiedades físicas lo que, por lo tanto, es muy eficiente para la detección de mercancía de contrabando. El sistema no solo mejora significativamente la eficiencia del despacho de carga, sino que también aumentará la precisión de la inspección, lo que reforzará la capacidad de combatir las actividades de contrabando en gran medida (HzMB, s.f.).

En lo que refiere al control aduanero, la apertura del HzM no solo proporciona un punto de acceso más para que los pasajeros se trasladen entre Hong Kong, China y Macao, sino que también acorta el tiempo de procesamiento de la carga transfronteriza para las industrias de logística. Para establecer una relación con la configuración de otros puntos de control y proporcionar un espacio sin contratiempos dentro de Guangdong-Hong Kong-Macao, las Aduanas de Hong Kong establecieron el "Contador de Servicio Prioritario AEo" y el "Espacio de Estacionamiento Prioritario AEO" en el punto de control del puente HZM. Al utilizar estas instalaciones designadas, las empresas AEo (certificadas como Operador Económico Autorizado por sus siglas en inglés) disfrutarán de un despacho de aduanas priorizado, así mismo, pueden ahorrar tiempo y costos en la gestión de su cadena de suministro (Gobierno de Hong Kong, 2018).

La sección del enlace ferroviario expreso Guangzhou-ShenzhenHong Kong y el puerto de Hong Kong del puente Hong Kong-ZhuhaiMacao comenzaron sus operaciones en septiembre y octubre del 2018, respectivamente. Al ser el primer proyecto de infraestructura transfronteriza que une los tres lugares, el Hzм no tiene precedentes. Se firmó un memorándum sobre los acuerdos de cooperación y asistencia mutua entre la Subadministración de Guangdong de la Administración General de Aduanas de la República Popular de China, el Departamento de Aduanas e Impuestos Especiales de Hong Kong y el Servicio de Aduanas de Macao para garantizar una aduana sin problemas de operación (Gobierno de Hong Kong, 2018).

De acuerdo con el reporte emitido por la Aduana de Hong Kong (2019), con la apertura del puente el lado oeste del delta del río Perla se 
incorpora a un radio de viaje de tres horas de Hong Kong. Disfrutando de la doble ventaja de la conectividad de tráfico brindada por el puente y las medidas de facilitación del comercio por parte de la Aduana de Hong Kong, los operadores de Hong Kong AEO están especialmente en una posición favorable para desarrollar su negocio. También se ha creado una situación de ganar-ganar tanto para la Aduana de Hong Kong como para el sector empresarial (Gobierno de Hong Kong, 2019).

El Plan de facilitación de transbordo intermodal de las Aduanas de Hong Kong instalado en el HzM está interconectado con el despacho aduanero rápido de las administraciones aduaneras de la provincia de Guangdong. Se han establecido un total de 63 puntos rápidos de despacho de carga en Hong Kong y en la provincia de Guangdong para acelerar el proceso de despacho de aduana de transbordo de carga entre los dos lugares, lo que pone a disposición 612 rutas de transbordo para los participantes del plan. Hasta finales de 2018, el esquema había procesado más de 6 millones de envíos de carga (Gobierno de Hong Kong, 2018).

En lo que respecta a los camiones de carga, aún pocos han utilizado el puente Hzм ya que las reglas de operación no se encuentran del todo claras y, por lo tanto, hay todavía poca carga de mercancías cruzada por este punto (Ma, 2019). De acuerdo con Ma (2019), tomando en cuenta información de la Autoridad del Puente HzM, solo 3429 vehículos que transportaban mercancías utilizaron el puente durante todo el mes de febrero del 2019. El número es muy inferior a 7020 en enero 2019 y 5302 en diciembre del 2018, aunque probablemente fue el resultado de las largas vacaciones durante el Festival de Primavera. Mientras tanto, la cantidad de autos privados aumentó de alrededor de 45000 por mes en diciembre y enero a 55117 en febrero (Ma, 2019).

\section{Conclusiones: el Puente HzM un parteaguas dentro de la BRI}

A lo largo de este artículo se ha enfatizado la importancia que tiene el binomio formado por la RPCH y las regiones de administración especial con respecto a esta magna obra, pues, más allá de la importancia que tiene Shanghai como eje de la vida económica dentro China continental, la región del delta del río Perla ha vivido un proceso histórico que le ha permitido consolidarse como un $h u b \mathrm{y}$, a partir de esta obra, como una zona de smart plugin debido al impacto comercial que genera. Sin 
duda alguna, la puesta en marcha del Puente HzM marca una nueva era en el comercio mundial, derivado de las tendencias tecnológicas aplicadas a la operatividad internacional, pues implica llevar un paso adelante los acuerdos y las facilitaciones al comercio a través de ingeniería innovadora que incrementa la competitividad de la megarregión. Con el funcionamiento del puente se prevé una expansión del volumen del comercio marítimo mundial del 3,2\% entre el 2017 y el 2022 (UNCTAD, 2017), aspecto que es vital ante el actual panorama mundial con respecto a la economía y la desaceleración de la economía china.

La innovación que implica el funcionamiento del puente HzM va más allá del aspecto tecnológico, pues deja una serie de lecciones a nivel geoeconómico, ya que esta obra se convierte en, o implica:

- Un proceso indirecto de integración económica que conjuga los intereses de las regiones de administración especial, desde la perspectiva de cooperación transfronteriza a través del CEPA, y la búsqueda hongkonesa de insertarse en la BRI. Aunque a nivel social y político ha sido compleja la implementación de esta iniciativa, desde su retorno a la $\mathrm{RPCH}$, la agenda económica conjunta y la infraestructura en el delta del río Perla ofrece un acercamiento profundo y vital para la sostenibilidad del modelo de crecimiento chino.

- Un mayor protagonismo del delta del río Perla, en particular de Hong Kong, en las actividades económicas de China a partir del papel que desempeña la infraestructura. Como se mencionó, la región es un modelo de smart plugin, lo que reinserta principalmente a Hong Kong dentro de la BRI, a través de la intermodalidad logística de impacto interno con las zEE y Macao, y externo con la conectividad hacia los corredores terrestres y marítimos de la BRI.

- Un hub de última generación en el sur de China, que haga contrapeso a la Zona de Libre Comercio (ZLC) ShanghaiPudong. En este aspecto, ya se ha señalado el tema del descongestionamiento del tráfico comercial como punto vital. Sin embargo, a diferencia de Shanghai, esta megarregión ofrece condiciones ventajosas en el contexto financiero. Si bien la ZLC Shanghai-Pudong ha impulsado iniciativas para un mayor posicionamiento financiero, es importante recordar que la gradualidad de la apertura no es tan grande como la que presenta 
Hong Kong. Esto se aúna a la certidumbre que genera un mercado financiero como el hongkonés, con reglas de operación más establecidas que un mercado como el shanghainés.

- Una estrategia de reposicionamiento de esta megarregión en términos de competitividad, principalmente como la puerta hacia el sureste asiático frente a puertos como Singapur, con el que existe una semejanza en términos de facilidad para los negocios y calidad de vida. Al mismo tiempo, se vuelve vital hacia un modelo de encadenamiento productivo de dos vías, en donde las regiones de administración especial, principalmente Hong Kong, se conviertan en el nodo central y estratégico:

Diagrama de relación y encadenamiento.

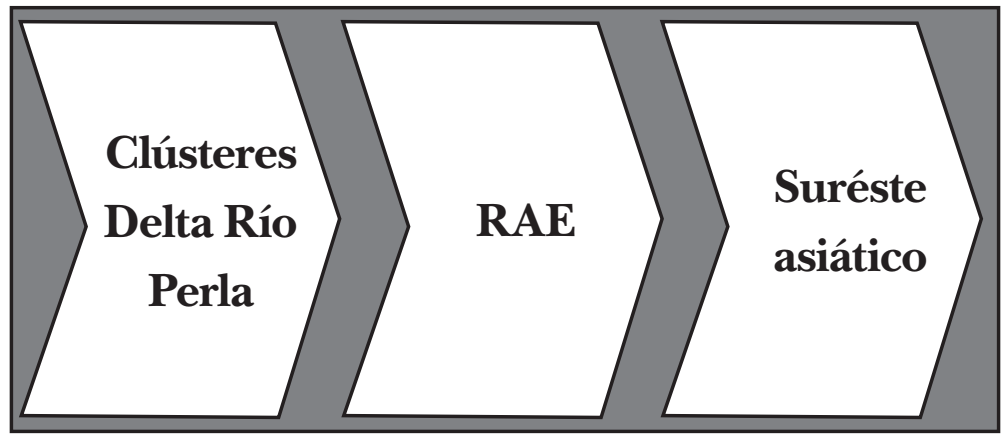

Fuente: Elaboración propia.

Ante todas estas lecciones, se enfatiza la importancia de la megarregión desde una perspectiva económica, pues sus características le permiten distinguirse de otras en el mundo. Es decir, la megarregión del delta del río Perla posee: acuerdos que facilitan el comercio, acceso a mano de obra especializada a partir de los clústeres y la ZNDII, e infraestructura. Por lo tanto, con relación a la geoeconomía, la construcción del puente deja ver que existe una estrategia económica por parte del gobierno chino que protege los intereses chinos a través del posicionamiento y por medio del incremento de la competitividad, así como a través del dominio de tecnologías clave como la infraestructura que le permite conquistar el mercado mundial (producción y comercialización) a través del modelo de negocios que permiten las condiciones actuales de las regiones de administración especial y la BRI. 
Finalmente, es importante destacar que, para que existan los beneficios descritos anteriormente, se debe generar una certidumbre basada en las reglas de operación para las autoridades y los usuarios de cualquier índole del HzM, debido a que, hasta el momento, aún no se cuenta con la información y facilitación necesaria para su uso óptimo.

\section{Referencias}

ACI. (2019). Annual Trafic Data 2017. Recuperado de https://aci.aero/data-centre/ annual-traffic-data/cargo/2017-cargo-summary-annual-traffic-data/

Azua, J. (2015). La ventaja competitiva de las naciones. Una experiencia de éxito, reorientando la estrategia transformadora del desarrollo económico y social del País Vasco. Cuadernos Orkestra, 2015(12), 3-9.

Cornejo, R. (1985). Las Zonas Económicas Especiales ¿Maquiladoras en China? Asia y África actuals, 20, 3(65), 444-469.

Chan, R. (2017). The Belt and Road Initiative: Opportunities for Developing Hong Kong's Infrastructure. 香港基建：對接「一帶一路」謀求發展新機遇. . Journal of Youth Studies, 20(1), 31-38.

Chen, H., y Unteroberdoerster, O. (2008). Hong Kong SAR Economic Integration with the Pearl River Delta (IMF Working Paper). Recuperado de: https://www.imf.org/ external/pubs/ft/wp/2008/wp08273.pdf https://doi.org/10.5089/9781451871319.001

Florida, R., Gulden, T., y Mellander, C. (2007). The Rise of the Mega-Region. Cambridge Journal of Regions, Economy Society, 2008(1), 459-476. https://doi.org/10.1093/cjres/rsn018

Gere, L., y Zoltai, A. (2018). The integration of the Pearl River Delta: The Hong Kong-Zhuhai-Macau Bridge. Pageo Geopolitika. Recuperado de http://www. geopolitika.hu/en/2018/08/22/the-integration-of-the-pearl-river-delta-thehong-kong-zhuhai-macau-bridge/\#_edn39

Gobierno de Hong Kong. (2017). Belt and Road, Hong Kong: a key link for the belt and road. Recuperado de https://www.beltandroad.gov.hk/compendium.pdf

Gobierno de Hong Kong. (2018a). AEO prioritized clearance facilities in the Hong Kong-Zhuhai-Macau Bridge (HZMB) control point. Recuperado de https://www.customs.gov.hk/en/trade_facilitation/aeo/update/publication/ index_20181121.html

Gobierno de Hong Kong. (2018b). Agreement on Trade in Goods signed under framework of CEPA. Recuperado de: https://www.info.gov.hk/gia/ general/201812/14/P2018121300670.htm

Gobierno de Hong Kong. (2019). Customs records vigorous law enforcement results and outlines smart development plan. Recuperado de https://www.customs. gov.hk/en/publication_press/press/index_id_2439.html

HSBC. (2016a). Pearl River Delta economy: an opportunity for all. Recuperado de: https://www.business.hsbc.uk/en-gb/how-china-is-shaping- 
international-trade-and-investment-flow/the-success-of-the-pearl-river-delta -economy

HSBC. (2016b). Pearl River Delta. Factbook. Recuperado de https://www.hsbc.com /-/files/hsbc-com/investorrelationsassets/presentationsandwebcasts/2016 /prd-factbook.pdf

HSBC. (2018). BRI impulsa el comercio exterior y crea oportunidades de negocio. Recuperado de https://www.empresas.hsbc.com.mx/es-mx/mx/article/ bri-impulsa-el-comercio-exterior-y-crea-oportunidades-de-negocio

HZMB. (s.f.). Hong Kong Port. Recuperado de https://www.hzmb.gov.hk/en/ project/hkbcf.html

ICEX. (Enero del 2010). Infraestructuras en Hong Kong. El exportador, 137.

Recuperado de http://www3.icex.es/icex/cda/controller/PageExportador/ 0,8723,6735394_6735502_6742676_4282019_4280226,00.html

KPMG. (2018). Hong Kong Banking Report 2018. Innovating for the future. Recuperado de https://assets.kpmg/content/dam/kpmg/cn/pdf/en/2018 /06/hong-kong-banking-report-2018.pdf

Kunaka, C. (2018). Six corridors of integration: Connectivity along the overland corridors of the Belt and Road Initiative.

Recuperado de http://blogs.worldbank.org/trade/six-corridors-integration -connectivity-along-overland-corridors-belt-and-road-initiative

Lee, R. (20 de enero del 2018). El puente de Hong Kong y la estrategia de globalización de China. Viento Sur. Recuperado de https://vientosur.info/ spip.php?article13411

Lin, Y. (s.f). The Development of the "Pan-Pearl River Delta Region" and the Interaction Between the Region and Taiwan.

Recuperado de: http://www.nira.or.jp/past/newsj/kanren/180/187/ pdf/E_lin.pdf

Liu, Q. (2011). Transformando el delta del río Perla. China Hoy.

Recuperado de: http://www.chinatoday.com.cn/ctspanish/se/txt/2011-03/16 /content_344128.htm

Lorot, P. (1999). Introducción a la Geoeconomía. París: Económica.

Luttwak, E. (1995). El sueño americano en peligro. París: O Jalcob.

Ma, J. (30 de marzo del 2019). Trucks aren't using Hong Kong-Zhuhai-Macau Bridge because rules are unclear, says Kerry Logistics chief George Yeo.

South China Morning Post. Recuperado de https://www.scmp.com/news/china /article/3003881/trucks-arent-using-hong-kong-macau-bridge-because -rules-are-unclear-says

Min, S. (2015). Pre-Colonial Southeast Asia and Tribute System. Tokio: Waseda University.

Nava, M. (2012). El Desarrollo Económico del Delta del Río Perla (Tesis de grado).

El Colegio de San Luis S.A., San Luis Potosí, México.

Recuperado de https://biblio.colsan.edu.mx/tesis

NavaHernandezMauricioManuel.pdf 
Papp, E. (2013). China-Africa: Zonas Económicas Especiales. Recuperado de http://www.politica-china.org/imxd/noticias/doc/1379142399China_y_las_ ZEEs_en_\%C3\%81frica.pdf

Reid, A. (1996) Flows and Seepages in the Long-Term Chinese Interaction with Southeast Asia. En A. Reid (ed.), Sojourners and Settlers: Histories of Southeast Asia and the Chinese (pp. 15-50). Australia: Allen \& Unwin.

Santander Trade. (2019). Hong Kong: Política y Economía. Recuperado de https:// es.portal.santandertrade.com/analizar-mercados/hong-kong/politicay-economia?\&actualiser_id_banque=oui\&id_banque=35\&memoriser_ choix $=$ memoriser

UNCTAD (2017). Informe sobre el transporte marítimo 2017 (Publicación de las Naciones Unidas). Recuperado de https://unctad.org/es/ PublicationsLibrary/rmt2017_es.pdf

Wang, H. (2016). A Deeper Look at China's "Going Out" Policy. Recuperado de https://www.cigionline.org/publications/deeper-look-chinas-going-out-policy

WEF. (2016). Competitive Cities and their Connections to Global Value Chains.

Recuperado de http://www.enovatinglab.com/archivos/descargas/

WhitePaper-GAC_Competitive_Cities_2016_06_17_12_34_21.pdf

WEF. (2018). China muestra al mundo el puente marítimo más largo del mundo:

55 kilómetros y acero para construir 60 torres Eiffel. Recuperado de https:// es.weforum.org/agenda/2018/02/china-muestra-al-mundo-el-puentemaritimo-mas-largo-del-mundo-55-kilometros-y-acero-para-construir60-torres-eiffel?utm_content=bufferea5a0\&utm_medium=social\&utm_ source=facebook.com\&utm_campaign=buffer

Wikipedia. (2007). Pearl River Delta Area. Recuperado de: https://es.m.wikipedia. org/wiki/Archivo:Pearl_River_Delta_Area.png

Zhou, M. (2013). China. Beijing: Foreign Languages Press. 